\title{
Temporally and spatially resolved investigation on the efflorescence process of a mixed droplet of ammonium sulfate and ammonium nitrate
}

\author{
WANG Feng ${ }^{1,2}$, ZHENG YuXia $^{1} \&$ ZHANG YunHong ${ }^{1 *}$ \\ ${ }^{1}$ The Institute of Chemical Physics, School of Science, Beijing Institute of Technology, Beijing 100081, China; \\ ${ }^{2}$ Chemistry Department, School of Physics and Chemistry, Henan Polytechnic University, Jiaozuo 454000, China
}

Received December 30, 2010; accepted March 30, 2011

\begin{abstract}
Temporally and spatially resolved investigations of the efflorescence of equimolar mixtures of ammonium sulfate (AS) and ammonium nitrate (AN) aerosol droplets were performed by confocal Raman spectroscopy combined with high-speed photography. Information was obtained on the dynamic process of morphological changes and the heterogeneous distribution of efflorescence products. The supersaturated AS/AN mixed droplet was found to first precipitate as the double salt 3AN.AS when the relative humidity was $50 \%$. The excess AS was irregularly distributed on the surface of the double salt as an inclusion structure. No formation of $2 \mathrm{AN} \cdot \mathrm{AS}$ was observed throughout the efflorescence process.
\end{abstract}

confocal Raman, high-speed photography, ammonium sulfate, ammonium nitrate, double salt

Citation: Wang F, Zheng Y X, Zhang Y H. Temporally and spatially resolved investigation on the efflorescence process of a mixed droplet of ammonium sulfate and ammonium nitrate. Chinese Sci Bull, 2011, 56: 2600-2603, doi: 10.1007/s11434-011-4617-6

Ammonium sulfate (AS) and ammonium nitrate (AN) are critical inorganic components in atmospheric aerosols [1]. Mixtures of compounds have also been shown to form double salt structures with various compositions in the aerosol during the efflorescence process. Martin and Schlenker et al. [2-4] investigated the efflorescence of $\mathrm{SO}_{4}{ }^{2-} / \mathrm{NO}_{3}{ }^{-} / \mathrm{H}^{+} / \mathrm{NH}_{4}{ }^{+}$ by Fourier transform infrared spectroscopy/aerosol flow tube (FTIR-AFT) analysis. They found two double salts, $3 \mathrm{AN} \cdot \mathrm{AS}$ and $2 \mathrm{AN} \cdot \mathrm{AS}$, formed along with pure AN and AS. The relative contents of these salts were dependant on the concentration ratio of AN/AS. Chan et al. [5] used an electrodynamic balance (EDB) in combination with Raman spectroscopy to study the efflorescence of equimolar AN/AS mixed in a single droplet, and found that the initial product of efflorescence was mainly metastable 3AN.AS. However, FTIR-AFT and EDB-Raman have some limitations and these studies have not provided in-depth descriptions on the spatial distribution of efflorescence products

*Corresponding author (email: yhz@bit.edu.cn) and its dynamic aspects. For FTIR-AFT, the statistical information is from a great number of aerosols droplets and it does not look at the differences in individual droplets, which makes it difficult to identify the mixing conditions of components such as the internal/external mixing states [6]. EDB-Raman has been widely applied for measurements of single particles. However, only the fundamental information of the droplet can be obtained and the growth status and spatial distribution of AN/AS in the mixed droplet throughout efflorescence process cannot be studied in detail. The confocal technique has high spatial resolution and has been applied to studies on aerosol particles with micro-scale spatial resolution [7,8]. A high-speed camera and optical microscope have been used in combination for a temporally resolved study on the morphological changes of aerosol droplets during efflorescence [9]. In the present study, confocal Raman spectroscopy was combined with high-speed photography for a temporally and spatially resolved study on the efflorescence of a mixed AS/AN droplet. This allowed monitoring of the dynamic process of morphological 
changes and the heterogeneous distribution of efflorescence products.

Equimolar mixtures of AN/AS solutions with a concentration of $0.5 \mathrm{~mol} / \mathrm{L}$ for each component was prepared and deposited on the quartz substrate using a micro syringe. The substrate was then placed in a sample cell. The relative humidity (RH) in the sample cell could be adjusted by controlling the flow rates of water vapor saturated with nitrogen and dry nitrogen gas into the cell. In situ monitoring of the morphological changes during the efflorescence process was achieved by high-speed photography (MS55K, MegaSpeed Corp, Canada). Confocal Raman spectroscopy (inVia, Renishaw, Britain) was used for spatial resolved analysis of the heterogeneous distribution of efflorescence products. The instrument settings and process have been described elsewhere $[8,9]$.

The Raman spectra of pure AN, AS, 3AN.AS and 2AN.AS crystals are shown in Figure 1. The spectra of pure $\mathrm{AN}$ and $\mathrm{AS}$ were acquired from the corresponding analytical grade chemicals, while the $3 \mathrm{AN} \cdot \mathrm{AS}$ and $2 \mathrm{AN} \cdot \mathrm{AS}$ for Raman analysis were obtained from the precipitants of different ratio mixtures of AN/AS solutions. These precipitants were screened to identify crystals that gave spectra consistent with those for 3AN-AS and 2AN-AS described in an earlier study [5]. In this earlier study, the Raman peaks of nitrate were located at 716 and $1045 \mathrm{~cm}^{-1}$, while those of sulfate were located at 451,614, and $975 \mathrm{~cm}^{-1}$. No distinct Raman peaks for ammonium were observed between 400 and $1100 \mathrm{~cm}^{-1}$ [5]. The nitrate peak at approximately 1050 $\mathrm{cm}^{-1}$ and the sulfate peak at $975 \mathrm{~cm}^{-1}$ could be clearly distinguished in the Raman spectra, and the peak position and shape were used in component identification and analysis for the AN/AS mixed droplet.

The time-dependent morphological changes of the AN/AS mixed droplet with a diameter of $100 \mu \mathrm{m}$ are presented in Figure 2. At 50\% RH, no obvious changes were observed in the droplet in the earlier stages. Metastable crystallization began to occur on the right edge of the droplet after $5 \mathrm{~min}$. For convenience, the moment that crystallization first took place was regarded as starting point. Following the appearance of the crystal seed, a number of

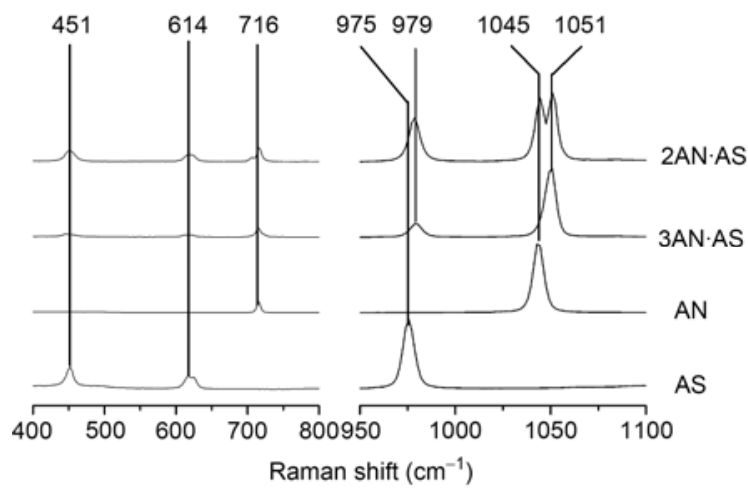

Figure 1 Raman spectra of pure AN, AS, 3AN·AS and 2AN·AS crystals. primary branches began to grow with time. Among these branches, two distinct branches had larger growth velocities and continuously grew until the whole droplet was covered by the crystal at $10.48 \mathrm{~s}$. The crystal growth was accompanied by obvious evaporation of water to equilibrate the humidity of the system, and this occurred throughout the crystal growth process. The final crystal morphology of the droplet is shown in the last picture in Figure 2.

To obtain an accurate image of the distribution of chemical compositions in the freshly formed crystal, the ambient RH was adjusted to $30 \%$, which suppressed the transformation of double salts from $3 \mathrm{AN} \cdot \mathrm{AS}$ to $2 \mathrm{AN} \cdot \mathrm{AS}$ [5]. Using micro-Raman spectroscopy, the Raman signals of the freshly formed crystal in different regions were acquired. Four typical regions marked as A, B, C and D were selected in reference to the dynamic images discussed above. Figure 3 presents the morphologies and Raman spectra of these different regions in the formed crystal. Regions A and B (Figure 3(a)) were located at the center and the edge of the largest branch, respectively. Region $\mathrm{C}$ was located at the edge of the region between the two large branches, and region $\mathrm{D}$ was located at the position where the crystallization first occurred. The Raman spectra at these four locations are shown in Figure 3(b). In the spectrum for region D, a strong peak appeared at $1051 \mathrm{~cm}^{-1}$ and a weak peak at $979 \mathrm{~cm}^{-1}$. Compared with the reference spectra (Figure 1), the crystal in region $\mathrm{D}$ was pure $3 \mathrm{AN} \cdot \mathrm{AS}$. For the same reasons, the crystal in region $\mathrm{C}$ could also be considered as $3 \mathrm{AN} \cdot \mathrm{AS}$ even though the signals were weak. In the spectrum of region $\mathrm{B}$, a strong peak at $1051 \mathrm{~cm}^{-1}$ indicated that $3 \mathrm{AN} \cdot \mathrm{AS}$ was present, and a peak at $975 \mathrm{~cm}^{-1}$ showed pure AS was present. The relatively weak intensity of the $975 \mathrm{~cm}^{-1}$ peak and its asymmetrical shape indicated that the content of pure AS was small. In the spectrum of region A, the crystal showed the highest purity of AS and only a small amount of 3AN.AS. The peak at $975 \mathrm{~cm}^{-1}$ was strong and almost symmetrical, and the peak at $1051 \mathrm{~cm}^{-1}$ was weak. Because of the low ambient RH, no characteristic peak for $2 \mathrm{AN} \cdot \mathrm{AS}$ was observed in any of the four regions or elsewhere (data not presented) for all the Raman spectra of the crystal.

In combination, the morphology and spatial-resolved

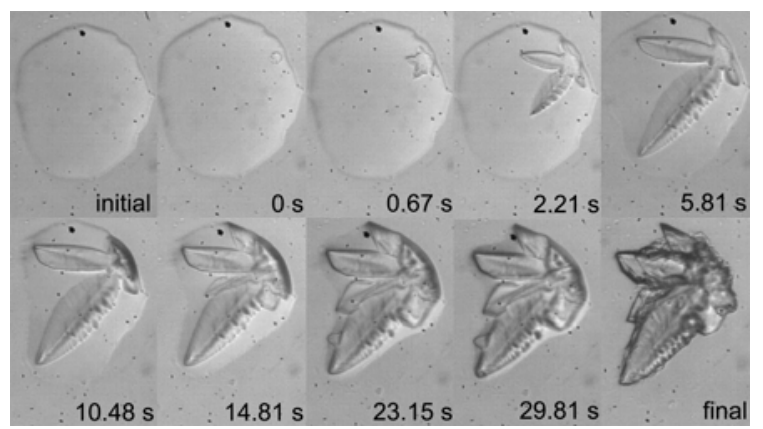

Figure 2 Time-dependent morphological changes of the equimolar AN/AS mixed droplet at a $\mathrm{RH}$ of $50 \%$. 

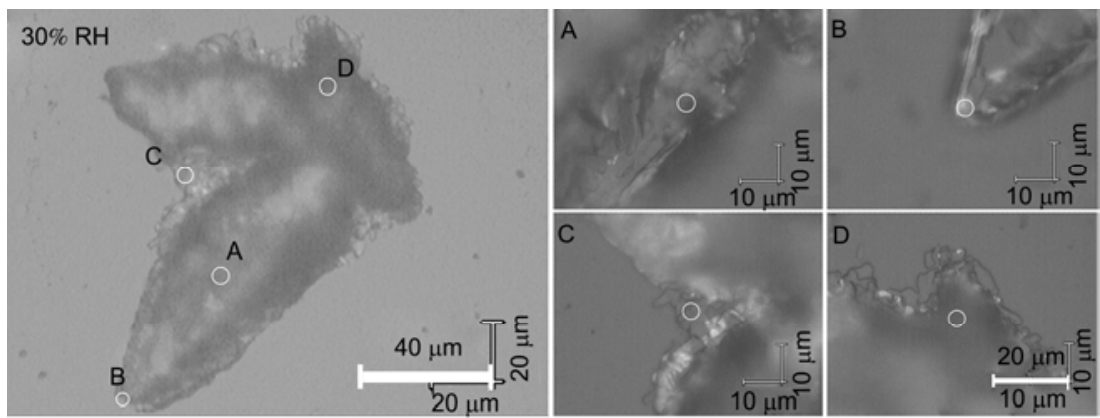

(a)

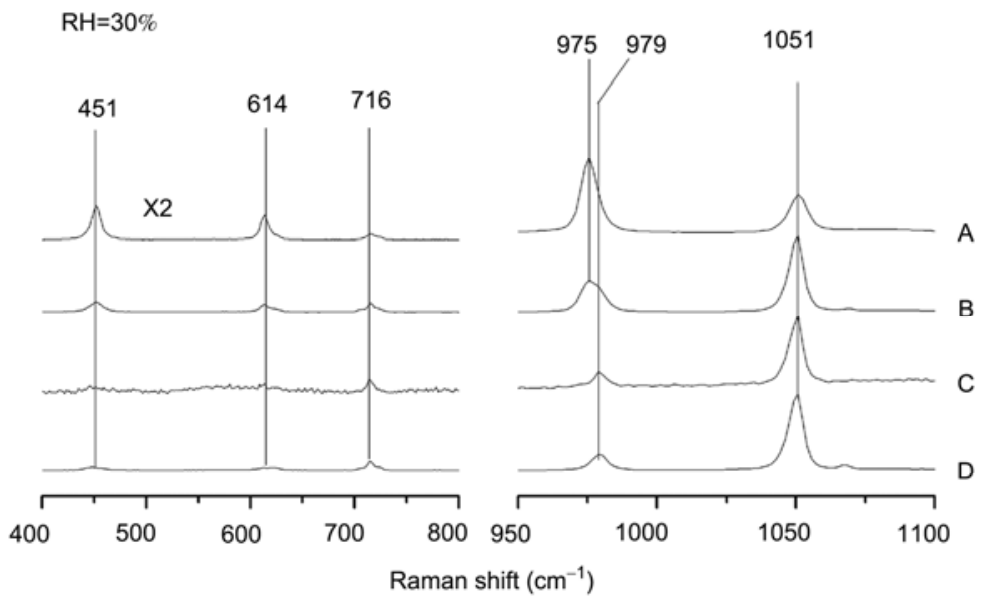

(b)

Figure 3 (a) Location and morphologies of efflorescence products in regions A, B, C and D; (b) Raman spectra of these regions.

Raman spectroscopy results can be used to deduce a reasonable mechanism for the crystallization process of an equimolar AN/AS mixed droplet. In this mechanism, the concentration of the droplet continues to increase and reaches supersaturation following the decrease in ambient humidity. As the RH reaches $50 \%$, metastable crystallization of 3AN.AS starts to occur on the edge of droplet. Accompanying water evaporation, results in the crystallization of $3 \mathrm{AN} \cdot \mathrm{AS}$ until the nitrate content remaining in the mixed droplet is negligible. The excess sulfate in the solution will then crystallize to form pure AS, which will be deposited irregularly on the surface of previously precipitated $3 \mathrm{AN} \cdot \mathrm{AS}$ to form an inclusion structure. Formation of 2AN.AS was not observed throughout the crystallization process.

Figure 4(a) and (b) shows the Raman spectra of the crystal at different depths in regions D and A, respectively. There was no change in the spectra in region D (Figure 4(a)) when the scanning depth was increased, which indicates that $3 \mathrm{AN} \cdot \mathrm{AS}$ was present at all depths and there were no other components. For region A (Figure 4(b)), the spectra showed the peak at $1051 \mathrm{~cm}^{-1}$ gradually increased compared with the peak at $975 \mathrm{~cm}^{-1}$, which suggests that the content of $3 \mathrm{AN} \cdot \mathrm{AS}$ increased gradually as the depth increased. The appearance of a $979 \mathrm{~cm}^{-1}$ shoulder on the peak at $975 \mathrm{~cm}^{-1}$ caused asymmetry, and provides further

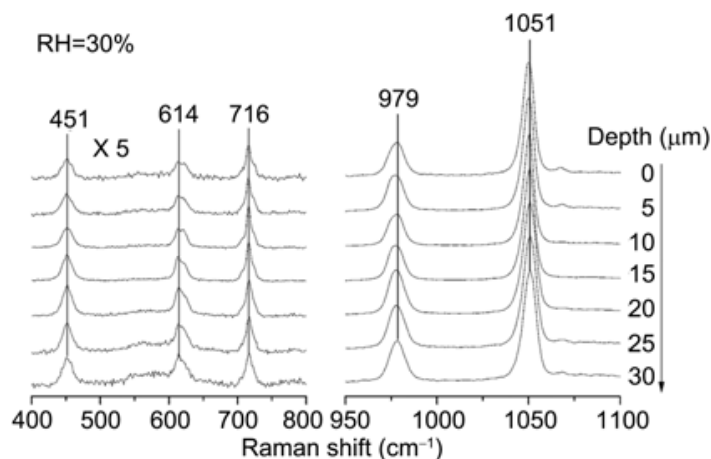

(a)

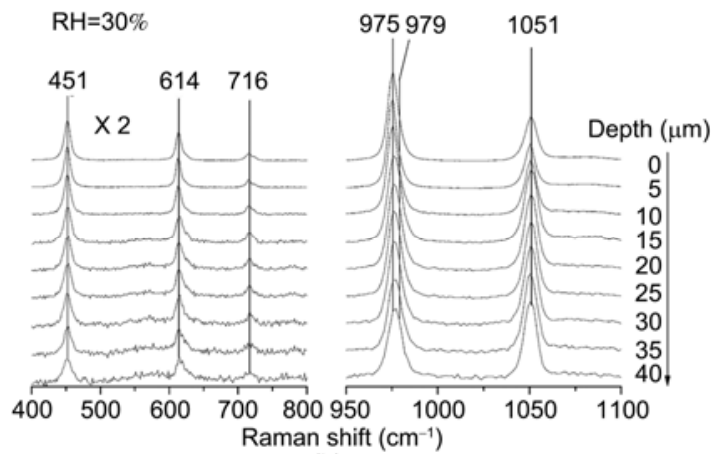

(b)

Figure 4 Raman spectra at various scanning depths for the efflorescence products of equimolar mixtures of AN/AS droplets at region D (a) and region $\mathrm{A}(\mathrm{b})$. 
evidence for this increase in $3 \mathrm{AN} \cdot \mathrm{AS}$.

The application of temporally and spatially resolved analysis of single particles of a multi-component aerosol droplet was useful to further understand the mechanisms of phase separation and transformation. The results can be used to evaluate the common principles of atmospheric aerosol phase transformation.

This work was supported by the National Natural Science Foundation of China (20973001 and 20903036).
1 Seinfeld J H, Pandis S N. Atmospheric Chemistry and Physics. New York: John Wiley and Sons Inc., 2006

2 Martin S T, Schlenker J C, Malinowski A, et al. Geophys Res Lett, 2003, 30: 2102

3 Schlenker J C, Malinowski A, Martin S T, et al. J Phys Chem A, 2004, 108: 9375-9383

4 Schlenker J C, Martin S T. J Phys Chem A, 2005, 109: 9980-9985

5 Ling T Y, Chan C K. Environ Sci Technol, 2007, 41: 8077-8083

6 Rosenoern T, Schlenker J C, Martin S T. J Phys Chem A, 2008, 112: 2378-2385

7 Everall N. Appl Spectrosc, 2008, 62: 591-598

8 Wang F, Shou J J, Zhang Y H. Chinese Sci Bull, 2008, 53: 2414-2416

9 Wang F, Zhao L J, Zhang Y H. Chinese Sci Bull, 2008, 53: 2140-2145

Open Access This article is distributed under the terms of the Creative Commons Attribution License which permits any use, distribution, and reproduction in any medium, provided the original author(s) and source are credited. 